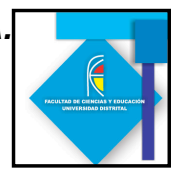

\title{
MEDICIÓN DEL CALOR LATENTE DE VAPORIZACIÓN DEL NITRÓGENO LÍQUIDO EN LA ENSEÑANZA DE LA FÍSICA EXPERIMENTAL
}

\begin{abstract}
MEASUREMENT OF THE LATENT HEAT OF VAPORIZATION LIQUID NITROGEN IN THE TEACHING OF EXPERIMENTAL PHYSICS
\end{abstract}

\author{
Miguel J. Espitia $\mathrm{R}^{1}$ \\ Ricardo E. Báez $C^{2}$ \\ Jhon H. Díaz $\mathrm{F}^{3}$
}

\section{Resumen}

Entre los métodos para la enseñanza de la física, uno de los más didáctico es el experimento, en este trabajo, se presenta una forma sencilla y económica para la medición indirecta del calor latente de vaporización del nitrógeno líquido utilizando elementos típicos de un laboratorio de física general, como son las resistencias, fuentes de voltajes, multímetros, cronómetros y una balanza, con ésta última se mide la variación de la masa en un recipiente que contiene nitrógeno liquido con respecto al tiempo, cuando se calienta el nitrógeno con una resistencia al hacer pasar por ésta una corriente eléctrica, encontramos una proporcionalidad entre la potencia eléctrica suministrada al nitrógeno, el tiempo y la masa de $\mathrm{N}_{2}$ evaporada, las cuales se relacionan con el calor latente de vaporización del nitrógeno.

Palabras clave: $\mathrm{N}_{2}$, calor latente, calor latente de vaporización, potencia eléctrica, bajas temperaturas.

\footnotetext{
Abstract

Among the methods for teaching physics, one of the more instructive is the experiment, we presents a simple and inexpensive for the indirect measurement of the latent heat of vaporization of liquid nitrogen using typical elements of a general physics laboratory, such as resistors, voltage sources, multimeters, timers and a balance, with the latter measures the mass change in a container of liquid nitrogen with respect to the time, when the nitrogen is heated with a resistance when it passes by an electric current, we found a

${ }^{1}$ Grupo NIDE, Facultad de Ingeniería, Universidad Distrital Francisco José de Caldas, Bogotá D. C. mjespitiar@yahoo.es

${ }^{2}$ Licenciatura en Física, Universidad Pedagógica Nacional, Bogotá D. C. dracir212@hotmail.com

${ }^{3}$ Grupo de Instrumentación Científica y Didáctica, Universidad Distrital Francisco José de Caldas, Bogotá.

jhdiazf@udistrital.edu.co
} 


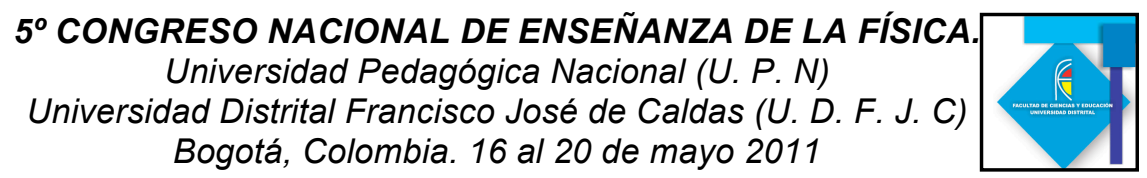

proportionality between the electric power supplied to nitrogen, time and the mass of Nitrogen evaporated, which relate to the latent heat of vaporization of nitrogen.

Keywords: $\mathrm{N}_{2}$, latent heat of vaporization, electrical power, low temperature

\section{Introducción}

Entre los métodos de la enseñanza de la física aquellos que utilizan el experimento son los más efectivos en el proceso de aprendizaje de un concepto ya que implica el trabajo directo del estudiante en la verificación de una ley o la medición de un observable físico. En este trabajo presentamos un método experimental sencillo y de bajo costo para la medición del calor latente de vaporización $L_{v}$ del nitrógeno a la presión atmosférica, utilizando la técnica denominada termogravimetría. Esta es una técnica experimental que se basa en la medición de la pérdida de masa producida por algún efecto térmico, como calentamiento de una resistencia eléctrica en interior de una masa de nitrógeno líquido en este caso y así determinar las cantidades de energía que se han utilizado en dicho proceso. La cantidad de energía (calor) liberada o absorbida por una sustancia durante una transición de fase se llama calor latente. Si a una determinada cantidad de sustancia añadimos calor continuamente se produce un cambio de fase de sólido a líquido y luego de líquido a vapor figura 1; estos cambios se llaman transiciones de fase y durante este proceso la temperatura del sistema no cambia. El calor latente absorbido por el nitrógeno líquido durante la transición líquido-vapor se llama calor latente de vaporización, esta energía térmica supera las fuerzas intermoleculares dentro del líquido lo que causa su separación .( Mahood, S. Anwar and W. Zia, 2009)

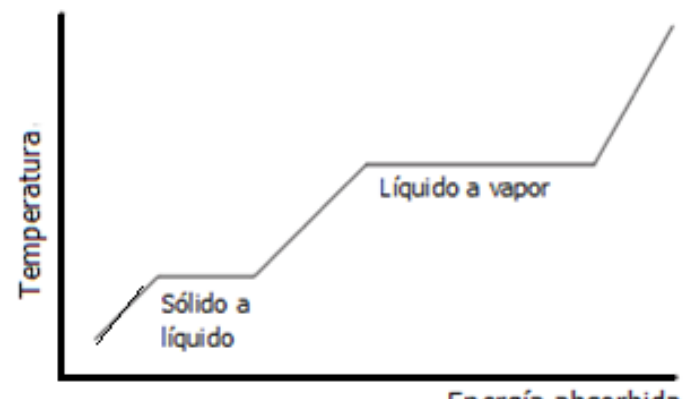

Figura 1. Cambio de fase cuando se agrega calor a un ritmo constante. La temperatura se mantiene constante durante la transición de fase y el calor suministrado en estos puntos se llama calor latente.

\section{Detalles experimentales}

La figura 1 muestra el diagrama simplificado del montaje experimental, mientras que la figura 3 se muestra el montaje real utilizado para la medición del calor latente de vaporización del Nitrógeno líquido. Un vaso de icopor que contiene Nitrógeno líquido se colocó en una balanza electrónica, una resistencia de R se colocó en el interior del vaso 


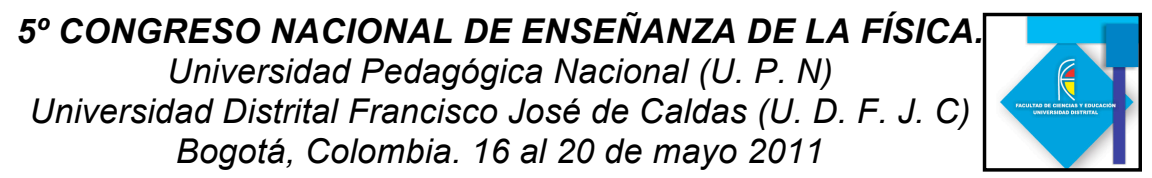

con $\mathrm{N}_{2}$ y se le suministró a dicha resistencia una diferencia de potencial $\mathrm{V}$ con el fin se suministrar calor al N2, se comenzó a contar el tiempo y a medir la pérdida de masa, cuando la masa de $\mathrm{N}_{2}$ en el vaso era de $200 \mathrm{gr}$.



Figura 2. Esquema simplificado del aparato utilizado para la medición de calor latente de vaporización Nitrógeno líquido, donde $B$ es la balanza, $C$ el vaso de icopor, $V$ el voltímetro, $\mathrm{R}$ la resistencia, $\mathrm{S}$ el interruptor.

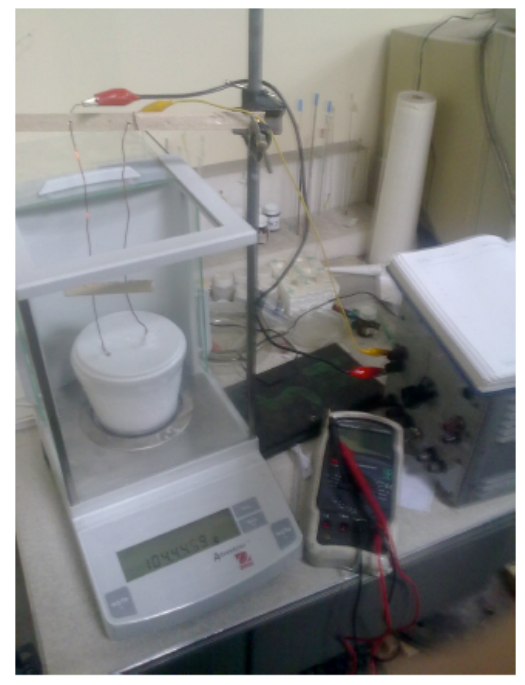

Fig. 4. Montaje experimental utilizado en la medición del Calor latente de vaporización del $\mathrm{N}_{2}$

La resistencia $\mathrm{R}$ que se introdujo en el vaso fue previamente sometida a un baño térmico con nitrógeno líquido con el fin de disminuir los efectos de un choque térmico al contacto con la muestra de $\mathrm{N}_{2}$ a analizar. Los resultados obtenidos se muestran en las tablas 1,2 y 3.

\section{Resultados}

Las tablas 01,02 y 03 muestran los resultados de la variación de la masa en función del tiempo para diferentes valores de resistencias eléctricas y distintos voltajes aplicados, observamos que a medida que transcurre el tiempo la evaporación de $\mathrm{N}_{2}$ aumenta a una razón aproximadamente constante. 


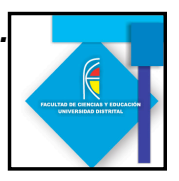

\begin{tabular}{|l|l|l|l|l|l|l|l|l|l|l|l|l|l}
\hline $\mathrm{m}(\mathrm{g})$ & 12.2 & 19.6 & 26.2 & 32.4 & 38.6 & 44.5 & 49.9 & 55.6 & 60.9 & 65.8 & 69.7 & 74.9 & 77.6 \\
\hline $\mathrm{t}(\mathrm{s})$ & 60.1 & 90.8 & 120.4 & 150.2 & 180.6 & 210.2 & 241.1 & 270.3 & 300.1 & 331.3 & 360.2 & 390.6 & 420.4 \\
\hline
\end{tabular}

Tabal 01: variación de la masa en función del tiempo $R=10 \Omega$ y $V=10.9 \mathrm{~V}$

\begin{tabular}{|l|l|l|l|l|l|l|l|l|l|l|l|l|}
\hline $\mathrm{m}(\mathrm{g})$ & 12.8 & 20.46 & 29.6 & 38.08 & 45.52 & 53.9 & 60.8 & 67.9 & 74.7 & 80.4 & 85.8 & 91.7 \\
\hline $\mathrm{t}(\mathrm{s})$ & 60.9 & 90.5 & 120.4 & 151 & 179.1 & 210.7 & 240.4 & 270.8 & 300.9 & 330.8 & 359.9 & 390.7 \\
\hline
\end{tabular}

Tabal 02: variación de la masa en función del tiempo $\mathrm{R}=10 \Omega$ y V $=21.6 \mathrm{~V}$

\begin{tabular}{|l|l|l|l|l|l|l|l|l|l|l|l|l|}
\hline $\mathrm{m}(\mathrm{g})$ & 10.1 & 20.2 & 30.6 & 40.1 & 48.6 & 58.2 & 68.3 & 77.0 & 87.6 & 96.0 & 105.5 & 114.2 \\
\hline $\mathrm{t}(\mathrm{s})$ & 60.4 & 90.7 & 120.5 & 150.6 & 180.6 & 210.7 & 240.4 & 270.6 & 299.8 & 330.4 & 360.0 & 390.6 \\
\hline
\end{tabular}

Tabal 03: variación de la masa en función del tiempo $\mathrm{R}=8 \Omega$ y V $=22.1 \mathrm{~V}$

La masa evaporada fue obtenida directamente de la lectura en la balanza electrónica, en cada caso antes de agregar el $\mathrm{N}_{2}$ en el vaso la lectura en la balanza se taró en $0 \mathrm{gr}$. El calor latente de Vaporización $\left(L_{v}\right)$ se define como la cantidad de calor $Q$ que hay que suministrar a un gramo de nitrógeno líquido a la temperatura de ebullición, $\mathrm{Te}=77 \mathrm{~K}$, para que pase al estado de vapor a la misma temperatura.

$$
L_{V}=\frac{\Delta Q}{m}
$$

Por otro lado, cuando circula una corriente constante I por una resistencia eléctrica $\mathrm{R}$ la potencia Joule es $P=I^{2} R$ y el calor disipado durante un intervalo de tiempo $\Delta t$ es:

$$
\Delta \in \mathbb{R} \pm \Delta \quad \frac{V^{2}}{R}
$$

Si esta es la única cantidad de calor que absorbe el líquido, se evapora una masa $m$ de la forma:

$$
\Delta € m L \quad V
$$

Entonces:

$$
m L t=\frac{V^{2}}{R}
$$

Por tanto:

$$
m(t)=\frac{\mathrm{A}^{2}}{R L_{V}}
$$

La figura 5A y 5B muestra la gráfica de la variación de la masa en función del tiempo correspondientes a las tablas 01 y 02 respectivamente, mientras que figura 6 muestra la 


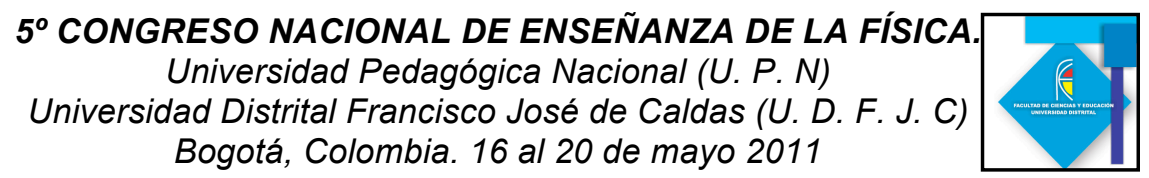

gráfica de la variación de la masa en función del tiempo correspondiente a la tabla 03, observamos que la masa de $\mathrm{N}_{2}$ evaporada aumenta linealmente con el tiempo, siendo la pendiente de dichas gráficas inversamente proporcional al calor latente de vaporización $L_{V}$ como se observa en la ecuación 5.

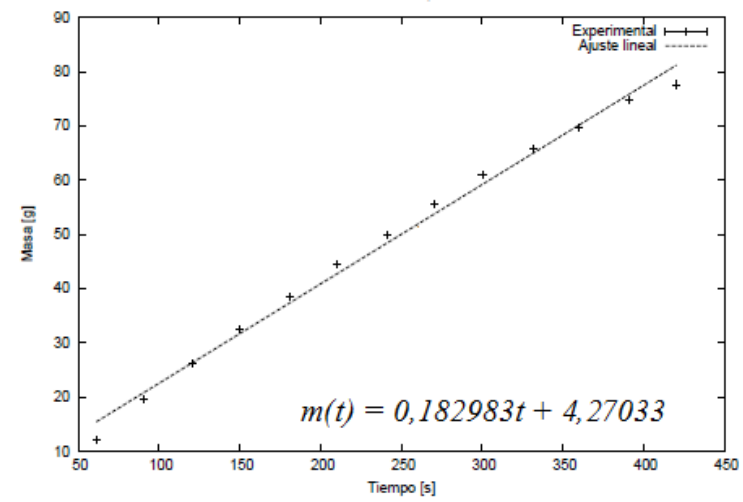

A

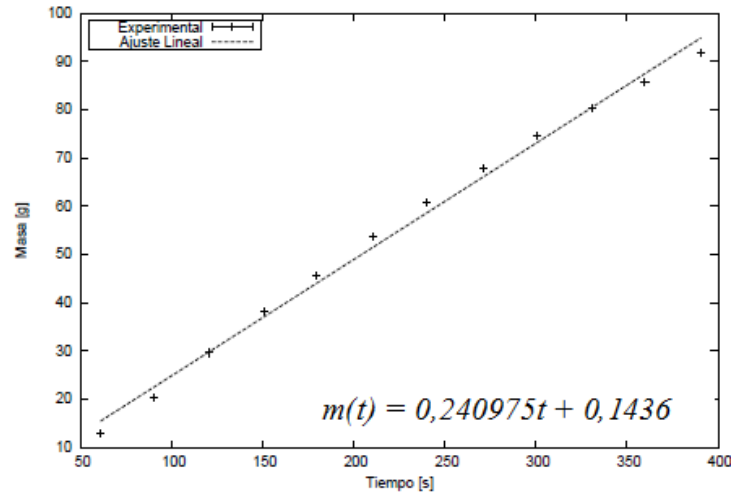

$B$

Figura 5. Variación de la masa en función del tiempo, A tabla 01 y B tabla 02.

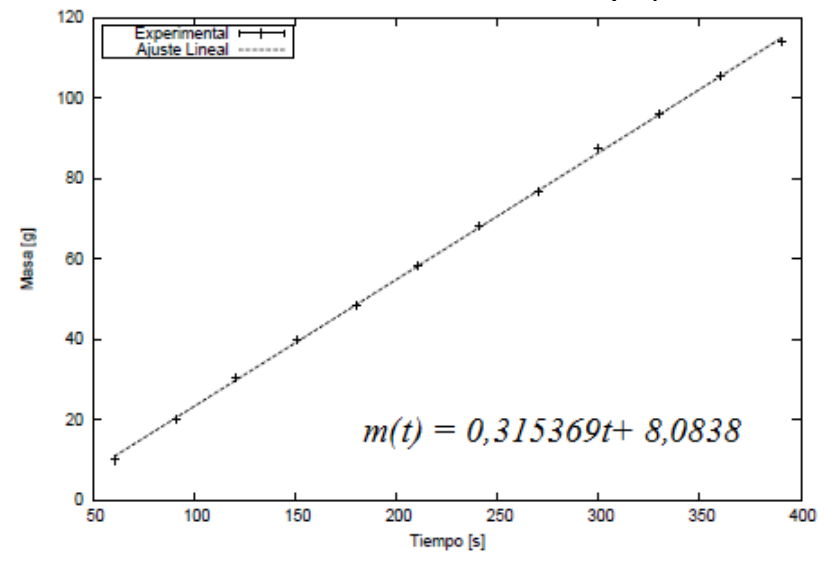

Figura 6. Variación de la masa en función del tiempo para la tabla 03

Mediante regresión lineal de los datos, se obtienen las ecuaciones de cada recta de cada tabla, las cuales se muestran en las figura 5 y 6 ; El valor 4,27033; 01,1436 y 8,0838 se asocian a la masa de nitrógeno líquido evaporada antes de introducir la resistencia calefactora en cada caso; por otro lado de acuerdo a la ecuación (5) la pendiente de cada recta viene dada por: $\mathrm{V}^{2} / \mathrm{RL}_{\mathrm{V}}$ y utilizando los valores de voltaje y resistencia en cada tabla obtuvimos un valor promedio para el calor latente de vaporización de: 194,14 J/gr con error relativo de $1,8 \%$ aproximadamente.

\section{Conclusiones}

El método experimental propuesto además de ser sencillo y de bajo costo es bastante efectivo en el cálculo del calor latente de vaporización del nitrógeno líquido, obtuvimos un valor de $194,14 \mathrm{~J} / \mathrm{gr}$ con error relativo de $1,8 \%$ aproximadamente. 


\section{$5^{\circ}$ CONGRESO NACIONAL DE ENSEÑANZA DE LA FÍSICA. \\ Universidad Pedagógica Nacional (U. P. N) \\ Universidad Distrital Francisco José de Caldas (U. D. F. J. C) \\ Bogotá, Colombia. 16 al 20 de mayo 2011

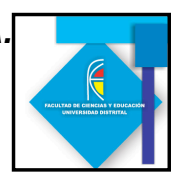

\section{Referencias Bibliográficas}

W. Mahood, S. Anwar and W. Zia. (2009), Latent heat of vaporization and specific Heat Capacity measurements using liquid nitrogen, LUMS School of Science and Engineering.

C.W. Tompson and H.W. White, (1982) Latent heat an low-temperature heat capacity experiment for the general physics laboratory, University of Missouri. 